\title{
Generalized scattering-matrix approach for magneto-optics in periodically patterned multilayer systems
}

\author{
B. Caballero, ${ }^{1,2}$ A. García-Martín, ${ }^{1}$ and J. C. Cuevas ${ }^{2,3}$ \\ ${ }^{1}$ IMM-Instituto de Microelectrónica de Madrid (CNM-CSIC), Isaac Newton 8, PTM, Tres Cantos, E-28760 Madrid, Spain \\ ${ }^{2}$ Departamento de Física Teórica de la Materia Condensada, Universidad Autónoma de Madrid, 28049 Madrid, Spain \\ ${ }^{3}$ Department of Physics, University of Konstanz, 78457 Konstanz, Germany
}

(Received 6 February 2012; revised manuscript received 27 March 2012; published 4 June 2012)

\begin{abstract}
We present here a generalization of the scattering-matrix approach for the description of the propagation of electromagnetic waves in nanostructured magneto-optical systems. Our formalism allows us to describe all the key magneto-optical effects in any configuration in periodically patterned multilayer structures. The method can also be applied to describe periodic multilayer systems comprising materials with any type of optical anisotropy. We illustrate the method with the analysis of a recent experiment in which the transverse magneto-optical Kerr effect was measured in an Fe film with a periodic array of subwavelength circular holes. We show, in agreement with the experiments, that the excitation of surface plasmon polaritons in this system leads to a resonant enhancement of the transverse magneto-optical Kerr effect.
\end{abstract}

DOI: 10.1103/PhysRevB.85.245103

PACS number(s): 78.20.Bh, 78.20.Ls, 78.66.Bz, 78.40.Kc

\section{INTRODUCTION}

In recent years a lot of attention has been paid to the study of the optical properties of nanostructured materials with both plasmonic and magneto-optic activity. ${ }^{1}$ The key idea is to use hybrid nanostructures containing both metals, which exhibit plasmon resonances, and ferromagnetic materials, which provide high magneto-optical activity for reasonably low values of the applied magnetic field, to profit from the best of the worlds of plasmonics and magneto-optics. In the context of these hybrid structures there are two main topics of interest. The first one is the use of the localization of the electromagnetic field due to the excitation of the plasmons supported by the free electrons in metals to enhance the magneto-optical signals (Kerr effect, Faraday effect, etc.). ${ }^{2,3}$ The nanostructuring in these magneto-plasmonic structures plays a fundamental role for several reasons. First of all, it provides a convenient way to couple the light of an external source to the plasmons supported by these hybrid systems, avoiding so the typical wave vector mismatch in unstructured systems. On the other hand, by nanostructuring these hybrid systems one can manipulate light at the nanometer scale in several ways. In particular, the enhancement of the electromagnetic field can be largely increased since one can concentrate light in reduced volumes. This can be done either by making use of localized plasmon resonances in isolated structures such as wires, ${ }^{3,4}$ disks, ${ }^{5-8}$ or particles, ${ }^{9,10}$ or by a periodic perforation in an otherwise continuous film. ${ }^{11-17}$

A second topic of interest is the use of magneto-optical effects to externally control either the properties of the transmission through perforated membranes ${ }^{18-21}$ or the very value of the surface plasmon wave vector. ${ }^{3,22-25}$ In this latter case, the relevant configuration is the transverse magneto-optical Kerr effect (see Sec. III below) since the other configurations induce a polarization conversion that implies a decoupling of the plasmon.

In view of the relevance of these novel hybrid structures, and in order to guide their design, it is crucial to have theoretical methods that are able to describe the wave propagation in nanostructured magneto-plasmonic systems. ${ }^{26-28} \mathrm{~A}$ powerful approach, which is widely used to describe nanostructured systems without magneto-optical activity, is the so-called scattering-matrix formalism. ${ }^{29-31}$ In recent years, this approach has been extended to study different magneto-optical effects in nanostructured multilayer structures ${ }^{32}$ and to describe the wave propagation in periodic structures containing certain types of anisotropic media. ${ }^{33}$ However, there are still basic physical situations which lie out of the scope of the existent implementations of the scattering formalism. Thus for instance, the Kerr and the Faraday effects in the transverse configuration, in which the magnetic field (or the magnetization of the sample) is parallel to the sample plane but perpendicular to the plane of incidence, cannot be addressed with the existent scattering-matrix-based approaches. More generally, when the optical anisotropy of the materials involves off-diagonal elements of the permittivity tensor along the growth direction of the multilayer structure, none of the existing implementations of the scattering-matrix approach can describe the wave propagation in such structures. The technical problem lies in the fact that in such situations the propagating eigenstates in the different layers cannot be simply described with a standard eigenvalue problem. In this work, we show how this problem can be solved and present a generalization of the scattering-matrix approach to describe the magneto-optics of hybrid nanostructured systems in any configuration. Moreover, the method can be applied to periodically patterned multilayer systems comprising any kind of optically anisotropic materials. We illustrate the capabilities of our formalism by addressing a recent experiment in which the transverse magneto-optical Kerr effect (TMOKE) was measured in a periodically perforated $\mathrm{Fe}$ film. ${ }^{14}$ We show that, in agreement with the experimental results, the excitation of surface plasmon polaritons in these structures leads to an enhancement of the TMOKE signal. More importantly, our theoretical method paves the way for studying a wide variety 
of plasmon-driven magneto-optical effects in periodic hybrid nanostructures.

The rest of the paper is organized as follows. In Sec. II we explain in detail how the scattering-matrix approach can be generalized to describe the wave propagation in all kind of magneto-optical and optically anisotropic periodic multilayer systems. Section III is devoted to the analysis of the experiments of Ref. 14, which allows us to illustrate the power of the method. Then, we shall summarize the main conclusions of our work in Sec. IV. Finally, several technical issues related to the formalism developed in Sec. II are addressed in detail in three appendixes.

\section{GENERALIZED SCATTERING-MATRIX APPROACH}

Our central goal is to solve the Maxwell's equations for a patterned multilayer structure containing any combination of materials (isotropic and anisotropic). For this purpose, we shall generalize the scattering-matrix approach developed by Whittaker and Culshaw in Ref. 29. Following this work, we shall first discuss the Maxwell's equations to be solved. Then, we shall address the band structure of an unbounded layer to determine the propagating eigenstates in the different layers. Then, we shall discuss how to construct the fields in a multilayer structure using those eigenstates and, finally we shall describe how the scattering matrix can be used to determine the field amplitudes in the whole structure.

\section{A. Maxwell's equations}

Let us start by describing the Maxwell's equations to be solved. Assuming a harmonic time dependence $\exp (-i \omega t)$, the Maxwell's equations adopt the following form: $\nabla \cdot \epsilon_{0} \bar{\epsilon} \mathbf{E}=0$, $\nabla \cdot \mathbf{H}=0, \nabla \times \mathbf{H}=-i \omega \epsilon_{0} \bar{\epsilon} \mathbf{E}$, and $\nabla \times \mathbf{E}=i \omega \mu_{0} \mathbf{H}$, where the permittivity is in general a tensor given by

$$
\bar{\epsilon}=\left(\begin{array}{ccc}
\epsilon_{x x} & \epsilon_{x y} & \epsilon_{x z} \\
\epsilon_{y x} & \epsilon_{y y} & \epsilon_{y z} \\
\epsilon_{z x} & \epsilon_{z y} & \epsilon_{z z}
\end{array}\right)
$$

Notice that we have assumed that $\bar{\mu}=\overline{1}$ since we are interested in the optical regime. Notice also that the first Maxwell's equation is automatically satisfied if the third one is fulfilled, and the second one can be satisfied by expanding the magnetic field in terms of basis functions with zero divergence. Following Ref. 29, we introduce the rescaling: $\omega \epsilon_{0} \mathbf{E} \rightarrow \mathbf{E}$ and $\sqrt{\mu_{0} \epsilon_{0}} \omega=\omega / c \rightarrow \omega$. Thus, the final two equations to be solved are

$$
\begin{aligned}
& \nabla \times \mathbf{H}=-i \bar{\epsilon} \mathbf{E} \\
& \nabla \times \mathbf{E}=i \omega^{2} \mathbf{H}
\end{aligned}
$$

We consider here multilayer systems in which each layer can be, in principle, periodically structured. Thus, the tensor $\bar{\epsilon}$ is independent of $z$, where $z$ corresponds to the growth direction of the structure, and it depends on the in-plane coordinates $\mathbf{r} \equiv(x, y)$ in a periodic fashion. Due to this periodicity, it is convenient to work in a momentum representation for the in-plane coordinates. Thus, for an in-plane wave vector $\mathbf{k}$, we can write the fields as a sum over reciprocal lattice vectors $\mathbf{G}$

$$
\begin{aligned}
& \mathbf{H}(\mathbf{r}, z)=\sum_{\mathbf{G}} \tilde{\mathbf{H}}_{\mathbf{k}}(\mathbf{G}, z) e^{i(\mathbf{k}+\mathbf{G}) \cdot \mathbf{r}}, \\
& \mathbf{E}(\mathbf{r}, z)=\sum_{\mathbf{G}} \tilde{\mathbf{E}}_{\mathbf{k}}(\mathbf{G}, z) e^{i(\mathbf{k}+\mathbf{G}) \cdot \mathbf{r}} .
\end{aligned}
$$

Now, it is convenient to define the Fourier space vectors ${ }^{29}$

$$
\begin{aligned}
\boldsymbol{h}(z) & \equiv\left[\tilde{\mathbf{H}}_{\mathbf{k}}\left(\mathbf{G}_{1}, z\right), \tilde{\mathbf{H}}_{\mathbf{k}}\left(\mathbf{G}_{2}, z\right), \ldots\right]^{T} \\
\boldsymbol{e}(z) & \equiv\left[\tilde{\mathbf{E}}_{\mathbf{k}}\left(\mathbf{G}_{1}, z\right), \tilde{\mathbf{E}}_{\mathbf{k}}\left(\mathbf{G}_{2}, z\right), \ldots\right]^{T} .
\end{aligned}
$$

Although $\tilde{\mathbf{H}}_{\mathbf{k}}$ and $\tilde{\mathbf{E}}_{\mathbf{k}}$ depend on $\mathbf{k}$, the calculation is done for a given value of $\mathbf{k}$ and therefore, we shall omit such labels in other quantities.

In what follows, we shall need the Fourier components of the permittivity tensor for the different layers

$$
\tilde{\epsilon}_{i j}(\mathbf{G})=\frac{1}{S} \int_{\text {unit cell }} d \mathbf{r} \epsilon_{i j}(\mathbf{r}) e^{-i \mathbf{G} \cdot \mathbf{r}},
$$

where $i, j=x, y, z, S$ is the area of the in-plane unit cell, and the matrix $\hat{\epsilon}_{i j}$ is such that $\left(\hat{\epsilon}_{i j}\right)_{\mathbf{G G}^{\prime}}=\tilde{\epsilon}_{i j}\left(\mathbf{G}-\mathbf{G}^{\prime}\right)$. Analogously, the components of the index tensor $\eta_{i j}(\mathbf{r})=\left[\bar{\epsilon}^{-1}(\mathbf{r})\right]_{i j}$ have Fourier expansions $\tilde{\eta}_{i j}(\mathbf{G})$ and matrix representations $\hat{\eta}_{i j}$.

With the notation just introduced, a product such as $\epsilon_{i j} \mathbf{E}$ becomes $\hat{\epsilon}_{i j} \boldsymbol{e}$ in momentum space. Thus, Eqs. (2) and (3) can be now written as

$$
\begin{gathered}
i \hat{k}_{y} h_{z}(z)-h_{y}^{\prime}(z)=-i \sum_{j} \hat{\epsilon}_{x j} e_{j}(z) \\
h_{x}^{\prime}(z)-i \hat{k}_{x} h_{z}(z)=-i \sum_{j} \hat{\epsilon}_{y j} e_{j}(z) \\
i \hat{k}_{x} h_{y}(z)-i \hat{k}_{y} h_{x}(z)=-i \sum_{j} \hat{\epsilon}_{z j} e_{j}(z),
\end{gathered}
$$

and

$$
\begin{gathered}
i \hat{k}_{y} e_{z}(z)-e_{y}^{\prime}(z)=i \omega^{2} h_{x}(z) \\
e_{x}^{\prime}(z)-i \hat{k}_{x} e_{z}(z)=i \omega^{2} h_{y}(z) \\
i \hat{k}_{x} e_{y}(z)-i \hat{k}_{y} e_{x}(z)=i \omega^{2} h_{z}(z),
\end{gathered}
$$

where $\hat{k}_{x}$ and $\hat{k}_{y}$ are diagonal matrices with $\left(\hat{k}_{x}\right)_{\mathbf{G G}}=\left(k_{x}+\right.$ $\left.G_{x}\right)$ and $\left(\hat{k}_{y}\right)_{\mathbf{G G}}=\left(k_{y}+G_{y}\right)$, and the primes stand for partial derivative with respect to $z$.

To conclude this subsection, let us say that matrices like $\hat{\epsilon}_{i j}$ or $\hat{\eta}_{i j}$ have in practice a finite dimension equal to $N_{G} \times N_{G}$, where $N_{G}$ is the number of reciprocal lattice vectors considered in the numerical calculations. It is also worth stressing that the simple Fourier factorization used above for the products like $\epsilon_{i j} E_{j}$, which is exact when $N_{G} \rightarrow \infty$, may lead in some cases to serious convergence problems when truncating the matrices $\hat{\epsilon}_{i j}$. The reason is that both the permittivity tensor and the electric field can exhibit discontinuities at the interfaces between different materials. The correct Fourier factorization of this type of products when $N_{G}$ is finite is discussed in detail in Appendix A.

\section{B. Band structure of a single layer}

Now our task is to solve the Maxwell's equations in momentum space derived in the previous subsection for the case of an unbounded layer. For this purpose, we expand the magnetic field in terms of $z$ propagating plane waves as 
follows: $:^{29}$

$$
\begin{aligned}
\mathbf{H}(\mathbf{r}, z)= & \sum_{\mathbf{G}}\left(\tilde{\phi}_{x}(\mathbf{G})\left[\hat{\mathbf{x}}-\frac{1}{q}\left(k_{x}+G_{x}\right) \hat{\mathbf{z}}\right]\right. \\
& \left.+\tilde{\phi}_{y}(\mathbf{G})\left[\hat{\mathbf{y}}-\frac{1}{q}\left(k_{y}+G_{y}\right) \hat{\mathbf{z}}\right]\right) e^{i(\mathbf{k}+\mathbf{G}) \cdot \mathbf{r}+i q z},
\end{aligned}
$$

where $\hat{\mathbf{x}}, \hat{\mathbf{y}}$, and $\hat{\mathbf{z}}$ are the Cartesian unit vectors and $q$ is the $z$ component of the wave vector. Here, $\tilde{\phi}_{x}(\mathbf{G})$ and $\tilde{\phi}_{y}(\mathbf{G})$ are the expansion coefficients to be determined by substituting into Maxwell's equations. Notice that this expression satisfies $\nabla$. $\mathbf{H}=0$. Now, it is convenient to rewrite the previous expression in momentum representation. By defining the vectors $\phi_{x}=$ $\left[\tilde{\phi}_{x}\left(\mathbf{G}_{\mathbf{1}}\right), \tilde{\phi}_{x}\left(\mathbf{G}_{\mathbf{2}}\right), \ldots\right]^{T}$ and $\phi_{y}=\left[\tilde{\phi}_{y}\left(\mathbf{G}_{\mathbf{1}}\right), \tilde{\phi}_{y}\left(\mathbf{G}_{\mathbf{2}}\right), \ldots\right]^{T}$, we can write

$$
\boldsymbol{h}(z)=e^{i q z}\left\{\phi_{x} \hat{\mathbf{x}}+\phi_{y} \hat{\mathbf{y}}-\frac{1}{q}\left(\hat{k}_{x} \phi_{x}+\hat{k}_{y} \phi_{y}\right) \hat{\mathbf{z}}\right\},
$$

where $\hat{k}_{x}$ and $\hat{k}_{y}$ are the diagonal matrices defined in Sec. II. For what follows, it is convenient to rewrite this last equation in the following vector notation:

$$
\boldsymbol{h}(z)=e^{i q z}\left(\phi_{x}, \phi_{y},-\frac{1}{q}\left(\hat{k}_{x} \phi_{x}+\hat{k}_{y} \phi_{y}\right)\right)^{T},
$$

where we recall that every entry in this column vector is a vector of dimension $N_{G}$. With this vector notation, Eqs. (9)(11) can now be written as

$$
\mathcal{C} \boldsymbol{h}(z)=\hat{\hat{\epsilon}} \boldsymbol{e}(z)
$$

where the block matrices $\mathcal{C}$ and $\hat{\hat{\epsilon}}$ are

$$
\mathcal{C}=\left(\begin{array}{ccc}
\hat{0} & q \hat{1} & -\hat{k}_{y} \\
-q \hat{1} & \hat{0} & \hat{k}_{x} \\
\hat{k}_{y} & -\hat{k}_{x} & \hat{0}
\end{array}\right), \quad \hat{\hat{\epsilon}}=\left(\begin{array}{ccc}
\hat{\epsilon}_{x x} & \hat{\epsilon}_{x y} & \hat{\epsilon}_{x z} \\
\hat{\epsilon}_{y x} & \hat{\epsilon}_{y y} & \hat{\epsilon}_{y z} \\
\hat{\epsilon}_{z x} & \hat{\epsilon}_{z y} & \hat{\epsilon}_{z z}
\end{array}\right) \text {. }
$$

On the other hand, Eqs. (12)-(14) adopt now the form

$$
\mathcal{C}^{T} \boldsymbol{e}(z)=\omega^{2} \boldsymbol{h}(z)
$$

From Eq. (18) we obtain the following expression for the electric field in momentum representation:

$$
\boldsymbol{e}(z)=\hat{\hat{\eta}} \boldsymbol{C h}(z),
$$

where $\hat{\hat{\eta}}=\hat{\epsilon}^{-1}$. Substituting this expression in Eq. (20) we obtain the following closed equation for the magnetic field in momentum representation:

$$
\mathcal{C}^{T} \hat{\hat{\eta}} \mathcal{C} \boldsymbol{h}(z)=\omega^{2} \boldsymbol{h}(z),
$$

which defines an eigenvalue problem for $\omega^{2}$. Indeed, only two of the three identities obtained from this equation, one for each $\hat{\mathbf{x}}, \hat{\mathbf{y}}$, and $\hat{\mathbf{z}}$, are independent. From the first two identities, and using Eq. (17), we obtain the following equations determining the allowed values for $q$ :

$$
\left(\mathcal{A}_{2} q^{2}+\mathcal{A}_{1} q+\mathcal{A}_{0}+\mathcal{A}_{-1} \frac{1}{q}\right) \phi=0,
$$

where $\phi=\left(\phi_{x}, \phi_{y}\right)^{T}$ and the $2 \times 2$ block matrices $\mathcal{A}_{n}$ are defined by

$$
\begin{aligned}
\mathcal{A}_{2} & =\left(\begin{array}{cc}
\hat{\eta}_{y y} & -\hat{\eta}_{y x} \\
-\hat{\eta}_{x y} & \hat{\eta}_{x x}
\end{array}\right), \\
\mathcal{A}_{1} & =\mathcal{A}_{1}^{(a)}+\mathcal{A}_{1}^{(b)}=\left(\begin{array}{cc}
-\hat{k}_{y} \hat{\eta}_{z y} & \hat{k}_{y} \hat{\eta}_{z x} \\
\hat{k}_{x} \hat{\eta}_{z y} & -\hat{k}_{x} \hat{\eta}_{z x}
\end{array}\right)+\left(\begin{array}{cc}
-\hat{\eta}_{y z} \hat{k}_{y} & \hat{\eta}_{y z} \hat{k}_{x} \\
\hat{\eta}_{x z} \hat{k}_{y} & -\hat{\eta}_{x z} \hat{k}_{x}
\end{array}\right), \\
\mathcal{A}_{0} & =\mathcal{A}_{0}^{(a)}+\mathcal{A}_{0}^{(b)}-\omega^{2} \hat{\mathbf{1}}=\left(\begin{array}{cc}
\hat{k}_{y} \hat{\eta}_{z z} \hat{k}_{y} & -\hat{k}_{y} \eta_{z z} \hat{k}_{x} \\
-\hat{k}_{x} \hat{\eta}_{z z} \hat{k}_{y} & \hat{k}_{x} \eta_{z z} \hat{k}_{x}
\end{array}\right)+\left(\begin{array}{cc}
\eta_{y y} \hat{k}_{x} \hat{k}_{x}-\eta_{y x} \hat{k}_{y} \hat{k}_{x} & \hat{\eta}_{y y} \hat{k}_{x} \hat{k}_{y}-\hat{\eta}_{y x} \hat{k}_{y} \hat{k}_{y} \\
\hat{\eta}_{x x} \hat{k}_{y} \hat{k}_{x}-\hat{\eta}_{x y} \hat{k}_{x} \hat{k}_{x} & \hat{\eta}_{x x} \hat{k}_{y} \hat{k}_{y}-\hat{\eta}_{x y} \hat{k}_{x} \hat{k}_{y}
\end{array}\right)-\omega^{2}\left(\begin{array}{cc}
1 & 0 \\
0 & 1
\end{array}\right), \\
\mathcal{A}_{-1} & =\left(\begin{array}{ll}
\hat{k}_{y} \hat{\eta}_{z x} \hat{k}_{y} \hat{k}_{x}-\hat{k}_{y} \hat{\eta}_{z y} \hat{k}_{x} \hat{k}_{x} & \hat{k}_{y} \hat{\eta}_{z x} \hat{k}_{y} \hat{k}_{y}-\hat{k}_{y} \hat{\eta}_{z y} \hat{k}_{x} \hat{k}_{y} \\
\hat{k}_{x} \hat{\eta}_{z y} \hat{k}_{x} \hat{k}_{x}-\hat{k}_{x} \hat{\eta}_{z x} \hat{k}_{y} \hat{k}_{x} & \hat{k}_{x} \hat{\eta}_{z y} \hat{k}_{x} \hat{k}_{y}-\hat{k}_{x} \hat{\eta}_{z x} \hat{k}_{y} \hat{k}_{y}
\end{array}\right) .
\end{aligned}
$$

In general, Eq. (23) is a so-called rational eigenvalue problem. This problem belongs to the category of nonlinear eigenvalue problems, which continues to be a challenge in the field of numerical analysis. However, we have found that a simple linearization strategy allows us to solve exactly such an eigenvalue problem in all the examples that we have studied. The details of this method are explained in Appendix B. It is worth stressing that so far the scattering approach has only been applied to situations where the materials are isotropic ${ }^{29}$ or in cases in which the magneto-optical activity is such that the off-diagonal components of the permittivity tensor involving the $z$ component are zero ${ }^{32}\left(\epsilon_{x z}=\epsilon_{y z}=0\right)$. In those cases, Eq. (23) reduces to

$$
\mathcal{A}_{0} \phi=-\mathcal{A}_{2} q^{2} \phi,
$$

which is a generalized eigenvalue problem for $q^{2}$, which can be solved with standard techniques of linear algebra. Notice that, as explained in the introduction, those cases exclude, for instance, the analysis of the Kerr effect in the transversal configuration.

The solution of Eq. (23) provides $4 N_{G}$ nonvanishing complex eigenvalues for $q$. Half of these eigenvalues lie in the upper half of the complex plane and half of them in the lower half. Finally, let us say that in the case of spatially uniform slabs, Eq. (23) reduces to a quartic equation for $q$, which is well known in the context of wave propagation in anisotropic media. This is shown in Appendix C.

\section{Electric and magnetic field}

The next step toward the complete solution of the Maxwell's equations in a multilayer structure is the determination of the fields in the different layers. This can be done by expressing the fields as a combination of forward and backward propagating 
waves with wave numbers $q_{n}$, and complex amplitudes $a_{n}$ and $b_{n}$, respectively. These amplitudes will be determined later on by using the boundary conditions at the interfaces and surfaces of the multilayer structure. Since the boundary conditions are simply the continuity of the in-plane field components, we focus here on the analysis of the field components $e_{x}, e_{y}, h_{x}$, and $h_{y}$. From Eq. (17), the in-plane components of $\boldsymbol{h}$ can be expanded in terms of propagating waves as follows:

$$
\left(\begin{array}{l}
h_{x}(z) \\
h_{y}(z)
\end{array}\right)=\sum_{n}\left\{\left(\begin{array}{c}
\phi_{x_{n}} \\
\phi_{y_{n}}
\end{array}\right) e^{i q_{n} z} a_{n}+\left(\begin{array}{c}
\varphi_{x_{n}} \\
\varphi_{y_{n}}
\end{array}\right) e^{-i p_{n}(d-z)} b_{n}\right\},
$$

where $d$ is the thickness of the layer. Here, $a_{n}$ is the coefficient of the forward going wave at the $z=0$ interface, and $b_{n}$ is the backward going wave at $z=d$. On the other hand, $q_{n}$ correspond to the eigenvalues of Eq. (23) with $\operatorname{Im}\left\{q_{n}\right\}>0$ and $p_{n}$ are the eigenvalues with $\operatorname{Im}\left\{p_{n}\right\}<0$. Notice also that, contrary to the case of isotropic materials, here the eigenfunctions of the forward and backward propagating waves are different in general. This is a sign of the fact that the existence of nonvanishing off-diagonal components of the permittivity tensor involving the $z$ coordinate can break the time-reversal symmetry in the system.

To make the notation more compact, we now define two $2 N_{G} \times 2 N_{G}$ matrices $\Phi_{+}$and $\Phi_{-}$whose columns are the vectors $\phi_{n}$ and $\varphi_{n}$, respectively. Moreover, we define the diagonal $2 N_{G} \times 2 N_{G}$ matrices $\hat{\mathrm{f}}_{+}(z)$ and $\hat{\mathrm{f}}_{-}(d-z)$, such that $\left[\hat{\mathrm{f}}_{+}(z)\right]_{n n}=e^{i q_{n} z}$ and $\left[\hat{\mathrm{f}}_{-}(d-z)\right]_{n n}=e^{-i p_{n}(d-z)}$, and the $2 N_{G}$-dimensional vectors $h_{\|}(z)=\left[h_{x}(z), h_{y}(z)\right]^{T}$, $a=\left(a_{1}, a_{2}, \ldots\right)^{T}$, and $b=\left(b_{1}, b_{2}, \ldots\right)^{T}$. In terms of these quantities, the in-plane magnetic-field components become

$$
h_{\|}(z)=\Phi_{+} \hat{\mathrm{f}}_{+}(z) a+\Phi_{-} \hat{\mathrm{f}}_{-}(d-z) b .
$$

Similarly, using the momentum representation of $\mathbf{E}$ from Eq. (21), it is straightforward to show that the in-plane components of the electric field $e_{\|}(z)=\left[-e_{y}(z), e_{x}(z)\right]^{T}$ are given by

$$
\begin{aligned}
e_{\|}(z)= & \left(\mathcal{A}_{0}^{(b)} \Phi_{+} \hat{q}^{-1}+\mathcal{A}_{1}^{(b)} \Phi_{+}+\mathcal{A}_{2} \Phi_{+} \hat{q}\right) \hat{\mathrm{f}}_{+}(z) a \\
& +\left(\mathcal{A}_{0}^{(b)} \Phi_{-} \hat{p}^{-1}+\mathcal{A}_{1}^{(b)} \Phi_{-}+\mathcal{A}_{2} \Phi_{-} \hat{p}\right) \hat{\mathrm{f}}_{-}(d-z) b,
\end{aligned}
$$

where the $\mathcal{A}$ 's are defined in Eq. (24) and we have defined the $2 N_{G} \times 2 N_{G}$ diagonal matrices $\hat{q}$ and $\hat{p}$ such that $\hat{q}_{n n}=q_{n}$ and $\hat{p}_{n n}=p_{n}$.

We can now combine Eqs. (27) and (28) into a single expression as follows:

$$
\begin{aligned}
\left(\begin{array}{l}
e_{\|}(z) \\
h_{\|}(z)
\end{array}\right) & =M\left(\begin{array}{c}
\hat{\mathrm{f}}_{+}(z) a \\
\hat{\mathrm{f}}_{-}(d-z) b
\end{array}\right) \\
& =\left(\begin{array}{ll}
M_{11} & M_{12} \\
M_{21} & M_{22}
\end{array}\right)\left(\begin{array}{c}
\hat{\mathrm{f}}_{+}(z) a \\
\hat{\mathrm{f}}_{-}(d-z) b
\end{array}\right),
\end{aligned}
$$

where the $2 N_{G} \times 2 N_{G}$ matrices $M_{i j}$ are defined as

$$
\begin{aligned}
& M_{11}=\mathcal{A}_{0}^{(b)} \Phi_{+} \hat{q}^{-1}+\mathcal{A}_{1}^{(b)} \Phi_{+}+\mathcal{A}_{2} \Phi_{+} \hat{q}, \\
& M_{12}=\mathcal{A}_{0}^{(b)} \Phi_{-} \hat{p}^{-1}+\mathcal{A}_{1}^{(b)} \Phi_{-}+\mathcal{A}_{2} \Phi_{-} \hat{p}, \\
& M_{21}=\Phi_{+}, \quad M_{22}=\Phi_{-} .
\end{aligned}
$$

\section{The scattering matrix}

The final step in our calculation is to use the scattering matrix ( $S$ matrix) to compute the field amplitudes needed to describe the different relevant physical quantities. This part of the calculation is practically independent of the type of materials present in the structure (isotropic or anisotropic), and it is nicely explained in Sec. V of Ref. 29. We just include a brief discussion here to make this work more self-contained.

By definition, the $S$ matrix relates the vectors of the amplitudes of forward and backward going waves $a_{l}$ and $b_{l}$, where $l$ now denotes the layer, in the different layers of the structure as follows:

$$
\left(\begin{array}{l}
a_{l} \\
b_{l^{\prime}}
\end{array}\right)=S\left(l^{\prime}, l\right)\left(\begin{array}{l}
a_{l^{\prime}} \\
b_{l}
\end{array}\right)=\left(\begin{array}{ll}
S_{11} & S_{12} \\
S_{21} & S_{22}
\end{array}\right)\left(\begin{array}{c}
a_{l^{\prime}} \\
b_{l}
\end{array}\right) .
$$

The field amplitudes in two consecutive layers are related via the boundary conditions for the fields, namely the continuity of the in-plane components of the fields in every interface and surface. If we consider the interface between the layer $l$ and the layer $l+1$, the corresponding boundary conditions read

$$
\left(\begin{array}{l}
e_{\|}\left(d_{l}\right) \\
h_{\|}\left(d_{l}\right)
\end{array}\right)_{l}=\left(\begin{array}{l}
e_{\|}(0) \\
h_{\|}(0)
\end{array}\right)_{l+1},
$$

where $d_{l}$ is the thickness of layer $l$. From this condition, together with Eq. (29), it is easy to show that the amplitudes in layers $l$ and $l+1$ are related by the interface matrix $I(l, l+1)=M_{l}^{-1} M_{l+1}$ in the following way:

$$
\begin{aligned}
\left(\begin{array}{c}
\hat{f}_{l}^{+} a_{l} \\
b_{l}
\end{array}\right) & =I(l, l+1)\left(\begin{array}{c}
a_{l+1} \\
\hat{f}_{l+1}^{-} b_{l+1}
\end{array}\right) \\
& =\left(\begin{array}{ll}
I_{11} & I_{12} \\
I_{21} & I_{22}
\end{array}\right)\left(\begin{array}{c}
a_{l+1} \\
\hat{f}_{l+1}^{-} b_{l+1}
\end{array}\right),
\end{aligned}
$$

where $\hat{f}_{l}^{+}=\hat{\mathrm{f}}_{l,+}\left(d_{l}\right)$ and $\hat{f}_{l+1}^{-}=\hat{\mathrm{f}}_{l+1,-}\left(d_{l+1}\right)$.

Now, with the help of the interface matrices, the $S$ matrix can be calculated in an iterative way as follows: The matrix $S\left(l^{\prime}, l+1\right)$ can be calculated from $S\left(l^{\prime}, l\right)$ using the definition of $S\left(l^{\prime}, l\right)$ in Eq. (31) and the interface matrix $I(l, l+1)$. Eliminating $a_{l}$ and $b_{l}$ we obtain the relation between $a_{l^{\prime}}, b_{l^{\prime}}$ and $a_{l+1}, b_{l+1}$, from which $S\left(l^{\prime}, l+1\right)$ can be constructed. This reasoning leads to the following iterative relations:

$$
\begin{aligned}
S_{11}\left(l^{\prime}, l+1\right)= & {\left[I_{11}-\hat{f}_{l}^{+} S_{12}\left(l^{\prime}, l\right) I_{21}\right]^{-1} \hat{f}_{l}^{+} S_{11}\left(l^{\prime}, l\right) } \\
S_{12}\left(l^{\prime}, l+1\right)= & {\left[I_{11}-\hat{f}_{l}^{+} S_{12}\left(l^{\prime}, l\right) I_{21}\right]^{-1} } \\
& \times\left(\hat{f}_{l}^{+} S_{12}\left(l^{\prime}, l\right) I_{22}-I_{12}\right) \hat{f}_{l+1}^{-} \\
S_{21}\left(l^{\prime}, l+1\right)= & S_{22}\left(l^{\prime}, l\right) I_{21} S_{11}\left(l^{\prime}, l+1\right)+S_{21}\left(l^{\prime}, l\right) \\
S_{22}\left(l^{\prime}, l+1\right)= & S_{22}\left(l^{\prime}, l\right) I_{21} S_{12}\left(l^{\prime}, l+1\right) \\
& +S_{22}\left(l^{\prime}, l\right) I_{22} \hat{f}_{l+1}^{-} .
\end{aligned}
$$

Starting from $S\left(l^{\prime}, l^{\prime}\right)=1$, one can apply the previous recursive relations to a layer at a time to build up $S\left(l^{\prime}, l\right)$.

From the knowledge of the $S$ matrix one can compute all the field amplitudes needed to describe a physical situation. Thus for instance, labeling the surface $l=0$ and the substrate $l=N$, the calculation of the reflectivity and the transmission coefficients requires the knowledge of the amplitudes $b_{0}$ and $a_{N}$, which can be calculated from $S(0, N)$. On the other hand, it may be interesting to calculate the fields inside the structure, 
for which we need the amplitudes $a_{l}$ and $b_{l}$. These can be obtained by calculating $S(0, l)$ and $S(l, N)$, and using Eq. (31) to get ${ }^{29}$

$$
\begin{aligned}
a_{l}= & {\left[1-S_{12}(0, l) S_{21}(l, N)\right]^{-1} } \\
& \times\left[S_{11}(0, l) a_{0}+S_{12}(0, l) S_{22}(l, N) b_{N}\right] \\
b_{l}= & {\left[1-S_{21}(l, N) S_{12}(0, l)\right]^{-1} } \\
& \times\left[S_{21}(l, N) S_{11}(0, l) a_{0}+S_{22}(l, N) b_{N}\right] .
\end{aligned}
$$

From these amplitudes, together with Eq. (29), one can determine the fields everywhere in the system, which usually provides an important physical insight into the different magneto-optical effects. ${ }^{2}$

\section{TMOKE IN PERFORATED IRON FILMS}

In this section we shall illustrate the method just described by analyzing the experiment reported in Ref. 14 in which the transverse magneto-optical Kerr effect (TMOKE) was studied in a periodically perforated $\mathrm{Fe}$ film. The TMOKE consists in an intensity change of the $p$ component of the reflected light upon application of a magnetic field perpendicular to the plane of incidence of the light. ${ }^{34}$ In the case of ferromagnetic materials, the magnetic field is used to reverse the magnetization $\mathbf{M}$ of the medium and the TMOKE is characterized by the following quantity

$$
\mathrm{TMOKE}=\frac{R_{p p}(+\mathbf{M})-R_{p p}(-\mathbf{M})}{R_{p p}(+\mathbf{M})+R_{p p}(-\mathbf{M})},
$$

where $R_{p p}( \pm \mathbf{M})$ are the reflectivity along the $p$ channel for the two opposite magnetizations, which in this configuration are perpendicular to the incidence plane and parallel to the layers of the structure. As explained in the introduction, this arrangement induces off-diagonal components of the permittivity tensor of the ferromagnetic material in the $z$ direction (direction of the growth of the multilayer) and therefore, it requires the use of the generalized scattering approach described in the previous section.

The structure studied in Ref. 14 is described schematically in Fig. 1. It consists of an Fe film (100 nm thick), which is perforated with a periodic array of subwavelength circular holes (diameter of $297 \mathrm{~nm}$ ) forming a triangular lattice with a lattice parameter of $470 \mathrm{~nm}$. The Fe film was prepared on a $\mathrm{Si}(111)$ substrate, and the structure contains additionally a seed layer of $\mathrm{Ti}$ ( $2 \mathrm{~nm}$ thick) and a capping layer of $\mathrm{Au}$ ( $2 \mathrm{~nm}$ thick), which were included to form a smooth Fe film and to prevent a subsequent oxidation of the surface, respectively. In our calculations we used the energy dependent permittivities taken from ellipsometric measurements of 20-nm-thick continuous films, and the off-diagonal elements of the ferromagnetic material have been extracted from Polar Kerr measurements (both rotation and ellipticity) as described in Ref. 35. Let us emphasize that in the case of the Fe film, the permittivity tensor in the transversal configuration described in Fig. 1(a) adopts the following form: ${ }^{34}$

$$
\bar{\epsilon}=\left(\begin{array}{ccc}
\epsilon & 0 & \epsilon_{x z} \\
0 & \epsilon & 0 \\
-\epsilon_{x z} & 0 & \epsilon
\end{array}\right),
$$

(a)
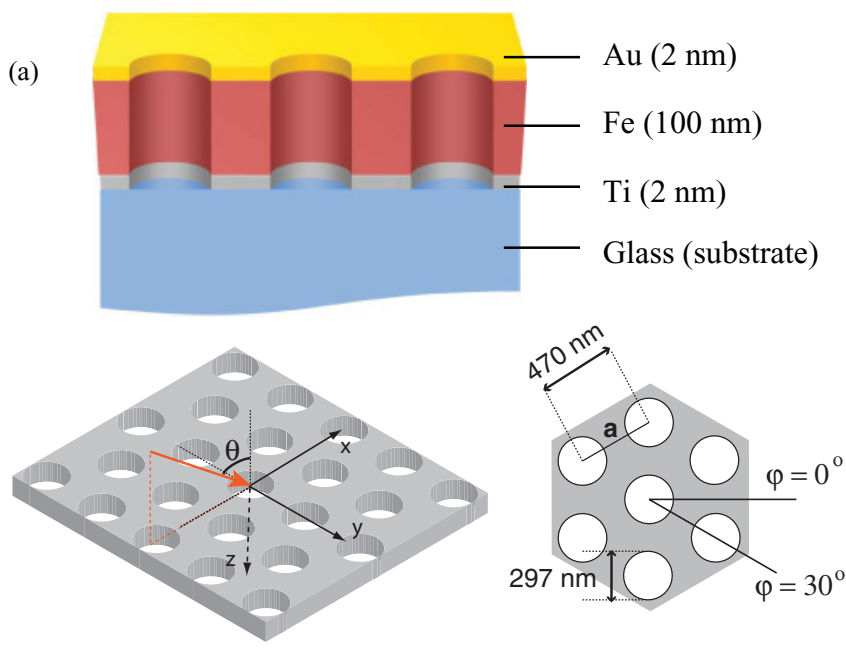

(b)

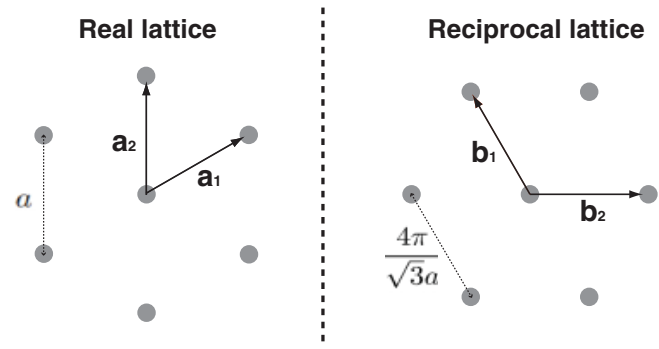

(c)
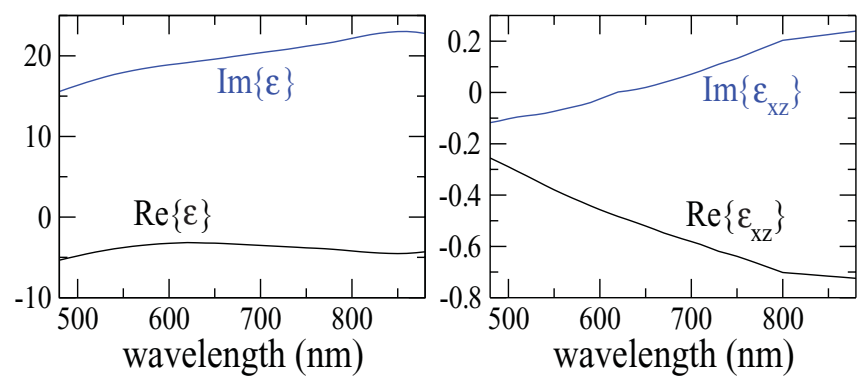

FIG. 1. (Color online) (a) Schematic representation of the system under study where we show a cut of the layered structure and a top view of the periodic array of circular holes forming a triangular lattice. Here, one can also see the angle definitions and the geometrical parameters of the hole array. (b) The triangular lattice both in real and in reciprocal space, and definition of the basis vectors. (c) The real and imaginary part of the elements $\epsilon$ (left panel) and $\epsilon_{x z}$ (right panel) of the Fe permittivity tensor used in our calculations, see Eq. (37), as a function of the wavelength.

where $\epsilon$ is the permittivity function of the nonmagnetized film and $\epsilon_{x z}=a M$, where $M$ is the magnitude of the magnetization at saturation. Let us recall that in the transverse configuration $\mathbf{M}=M \hat{\mathbf{y}}$, i.e., the magnetization is parallel to the Fe film and perpendicular to the plane of incidence. For completeness, we show in Fig. 1(c) the values of $\epsilon$ and $\epsilon_{x z}$ for Fe used in our calculations as a function of the wavelength. Notice that the magnitude of $\epsilon_{x z}$ is much smaller than the magnitude of $\epsilon$, which explains the "smallness" of the different magnetooptical effects.

Since the holes of our structure are circular, the Fourier expansion of the permittivity, Eq. (8), can be calculated analytically. ${ }^{36}$ For holes of radius $r$ and permittivity 


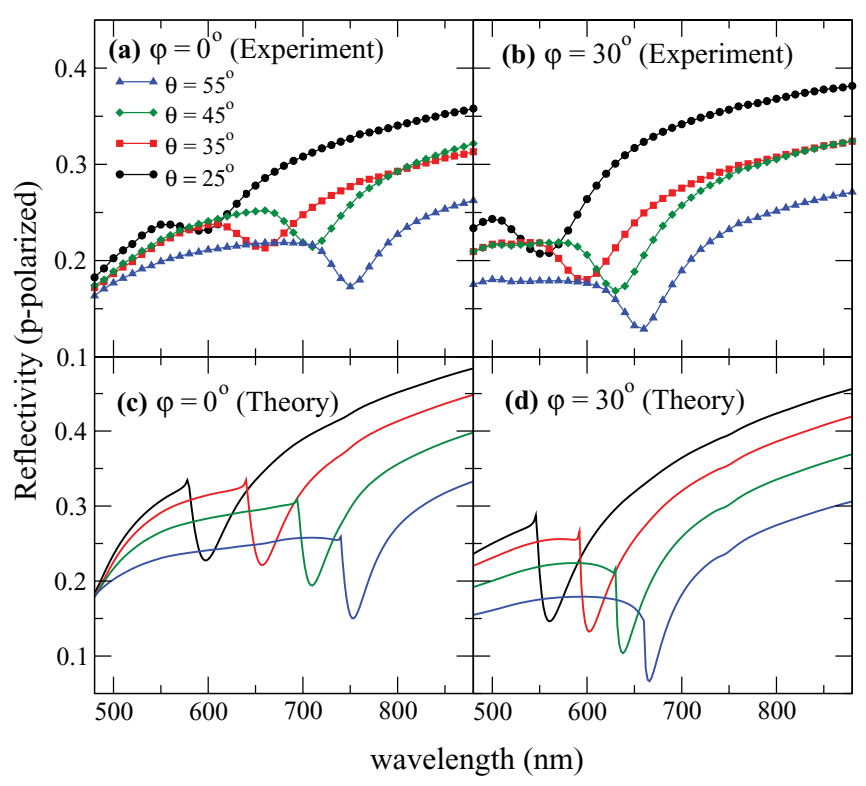

FIG. 2. (Color online) Experimental and theoretical results for the reflectivity of the demagnetized structure along the $p$ channel $R_{p p}$ as a function of the wavelength of the incident light. As indicated in the panels, the results are shown for two different high symmetry crystallographic directions $\varphi=0^{\circ}$ and $\varphi=30^{\circ}$ and for various angles of incidence $\theta$.

components $\epsilon_{i j}^{h}$ in a material with permittivity $\epsilon_{i j}^{m}$, we have

$$
\tilde{\epsilon}_{i j}(\mathbf{G})= \begin{cases}2\left(\epsilon_{i j}^{h}-\epsilon_{i j}^{m}\right) \beta J_{1}(G r) /(G r) & \text { if } \mathbf{G} \neq 0 \\ \epsilon_{i j}^{m}+\beta\left(\epsilon_{i j}^{h}-\epsilon_{i j}^{m}\right) & \text { if } \mathbf{G}=0\end{cases}
$$

where $\beta$ is the fraction of the area occupied by the holes, and $J_{1}$ is a Bessel function of the first kind. In the case of a triangular lattice with lattice constant $a_{0}, \beta=(2 / \sqrt{3}) \pi r^{2} / a_{0}^{2}$.

In the numerical calculations performed to obtain the results that we are about to describe we have truncated the Maxwell equations by setting a high-momentum cutoff, and we have employed the fast Fourier factorization described in Appendix A. In particular, the results shown in what follows were obtained by using $N_{G}=367$ lattice vectors, which suffices to converge the different physical properties discussed here (see Appendix A).

Let us start our discussion of the results by describing the reflectivity in this multilayer system when the Fe film is demagnetized. In the upper panels of Fig. 2 we reproduce the experimental results for $p$-polarized light obtained for two different high symmetry crystallographic directions $\varphi=0^{\circ}$ and $\varphi=30^{\circ}$ and for various angles of incidence $\theta$ (see Fig. 1(a) for a definition of these angles). ${ }^{37}$ The most prominent feature is the appearance of a dip which is red shifted as the angle of incidence $\theta$ is increased. Notice that the red shift depends on the crystallographic direction, and it is more pronounced for $\varphi=0^{\circ}$. Such a feature is absent in the case of $s$-polarized light (not shown here), and it can be attributed to the excitation of surface plasmon polaritons (SPPs), as we shall discuss below. In the lower panel of Fig. 2, we show the corresponding theoretical results calculated with the scattering approach assuming that $\epsilon_{x z}$ in Eq. (37) is zero. As one can see, our calculations nicely reproduce the experimental

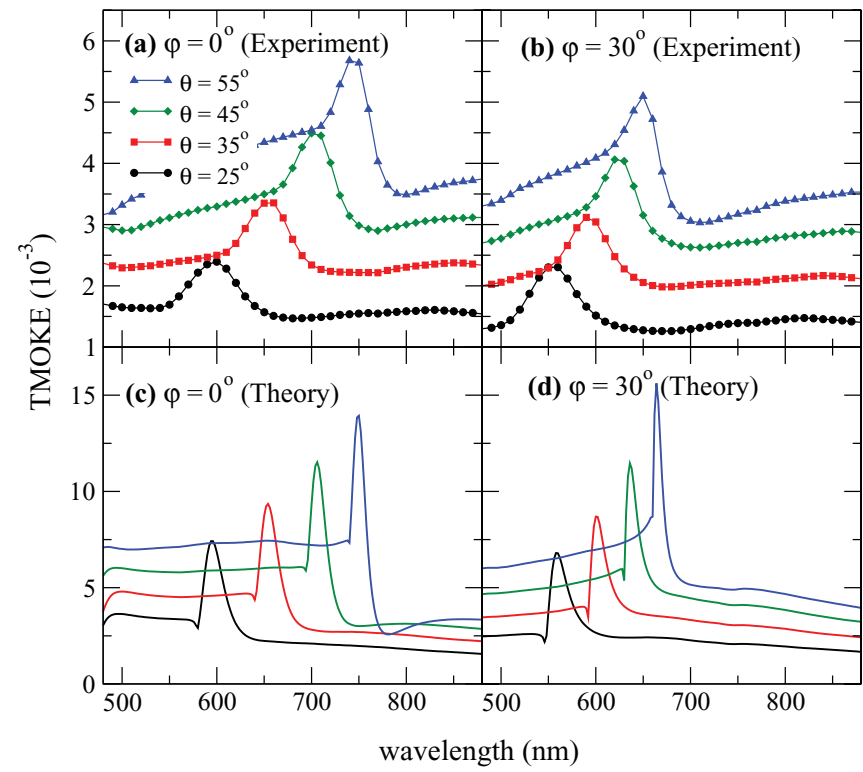

FIG. 3. (Color online) Experimental and theoretical results for the TMOKE as a function of wavelength for $\varphi=0^{\circ}$ and $\varphi=30^{\circ}$ and for various angles of incidence $\theta$.

trends. The theoretical dips appear to be more pronounced than in the experiment, which we attribute to the unavoidable inhomogeneities in the periodic array of holes in the Fe film.

The corresponding results (both experimental and theoretical) for the TMOKE, as defined in Eq. (36), are displayed in Fig. 3. Notice that the theoretical results, in good agreement with the experiment, show that the TMOKE can be resonantly enhanced at wavelengths that follow closely those in which the dips in the reflectivity appear. Notice that at resonance the TMOKE signal increases by roughly a factor of 2 with respect to the value at off-resonant wavelengths. It is worth stressing that, as illustrated in Fig. 4, the signal for the continuous Fe film (not perforated) is featureless in the spectral range considered here.

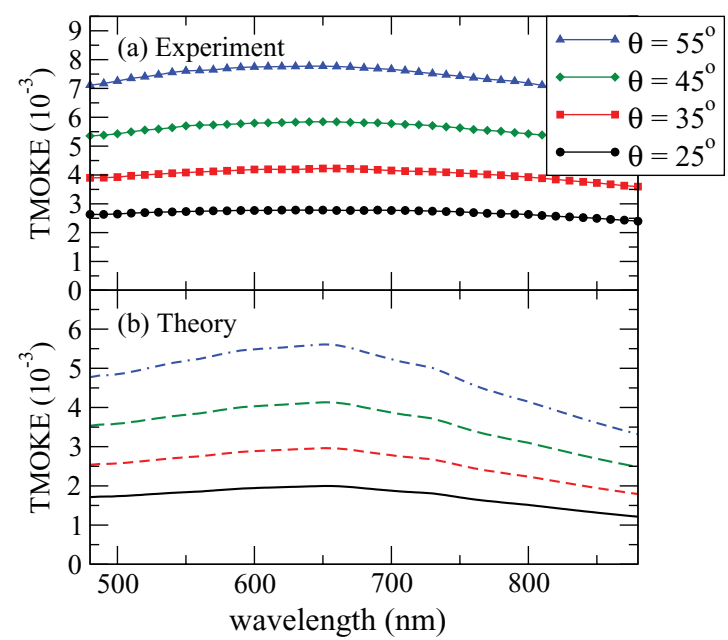

FIG. 4. (Color online) (a) Experimental results for the TMOKE for the nonperforated multilayer structure (formed by uniform slabs) as a function of wavelength for various angles of incidence $\theta$ taken from Ref. 14. (b) The corresponding theoretical results. 

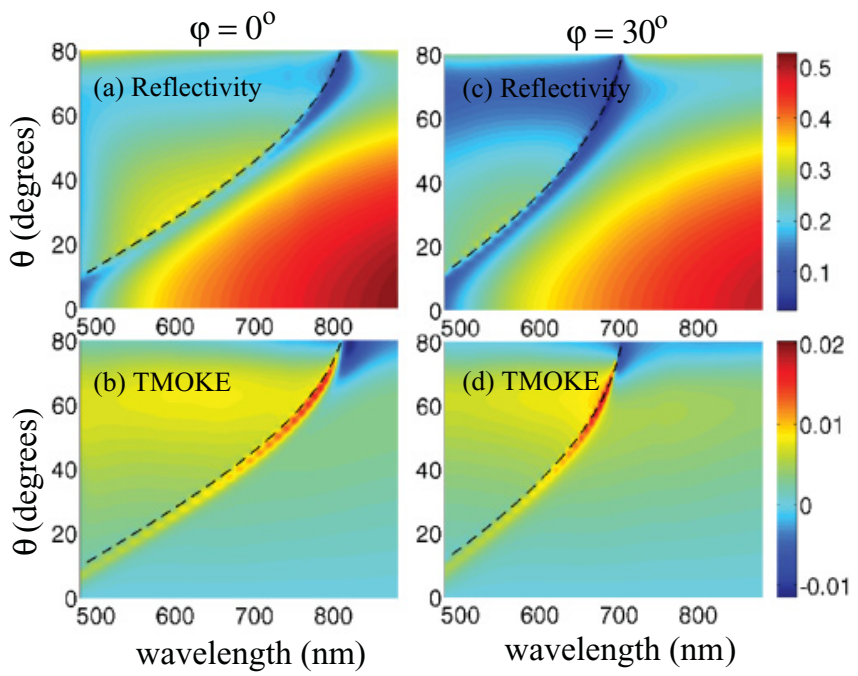

FIG. 5. (Color online) Theoretical results for the reflectivity and TMOKE as a function of the wavelength and angle of incidence $\theta$ for $\varphi=0^{\circ}$ and $\varphi=30^{\circ}$. The dashed lines in the different panels correspond to the resonant condition for the excitation of SPPs, as described by Eq. (40).

In order to understand the origin of the peaks in the TMOKE and the corresponding dips in the reflectivity, we have investigated these quantities in a more systematic way. In Fig. 5 we present the results for these two quantities as a function of the wavelength and of the angle of incidence $\theta$. In this figure we can observe again the appearance of the dips in the reflectivity, which are accompanied by pronounced peaks in the TMOKE. The shape of the TMOKE and the dispersion of the peaks with $\theta$ suggest that these features originate from the excitation of the SPPs of this structure. To confirm this impression we have to calculate the matching condition for the excitation of these surface modes. For this purpose, we need first to determine the dispersion relation of the SPPs and, as an approximation, we shall assume that it is given by the dispersion relation for continuous films. Thus, ignoring the thin Au layer, which is practically transparent, the complex wave vector of the SPP modes is given by ${ }^{38}$

$$
k_{\mathrm{spp}}(\lambda)=\frac{2 \pi}{\lambda} \sqrt{\frac{\epsilon(\lambda)}{1+\epsilon(\lambda)}},
$$

where $\lambda$ is the light wavelength, $\epsilon$ is the permittivity of the demagnetized $\mathrm{Fe}$ film, and we have used the fact that the incidence medium is air. Now, the matching condition, which is the conservation of the parallel wave vector, can be written as

$$
\left|\operatorname{Re}\left\{k_{\mathrm{spp}}\left(\lambda_{n_{1}, n_{2}}\right)\right\}\right|=\left|\mathbf{k}_{\|}+\mathbf{G}_{n_{1}, n_{2}}\right|,
$$

where $\mathbf{k}_{\|}=(2 \pi / \lambda) \sin \theta \hat{\mathbf{x}}$ is the in-plane wave vector of the incoming light [in the transverse configuration described in Fig. 1(a)] and $\mathbf{G}_{n_{1} n_{2}}$ is a reciprocal lattice vector, which with our choice for the reciprocal lattice basis vectors [see Fig. $1(\mathrm{~b})]$ is given by $\mathbf{G}_{n_{1}, n_{2}}=\left(2 \pi / a_{0} \sqrt{3}\right)\left\{\left[\left(2 n_{2}-n_{1}\right)\right.\right.$ $\left.\left.\cos \varphi+n_{1} \sqrt{3} \sin \varphi\right] \hat{\mathbf{x}}+\left[n_{1} \sqrt{3} \cos \varphi+\left(n_{1}-2 n_{2}\right) \sin \varphi\right] \hat{\mathbf{y}}\right\}$. The condition of Eq. (40) tells us at which (discrete) wavelengths the SPPs can be excited for a given angle of incidence. We have solved Eq. (40) numerically and found that for $\varphi=0^{\circ}$ the only mode that can be excited in the wavelength range analyzed here is $\lambda_{0,-1}$, while for $\varphi=30^{\circ}$ we have two possibilities: $\lambda_{0,-1}$ and $\lambda_{-1,-1}$, which indeed correspond to the same wavelength. In Fig. 5 we have included as dashed lines the relation between the resonant wavelength and the angle of incidence $\theta$ for these two cases. As one can see, these relations nicely describe the positions of both the dips in the reflectivity and the peaks in the TMOKE. This strongly suggests that the resonant enhancement of the TMOKE is due to the excitation of SPPs in our layered structure.

\section{CONCLUSIONS}

We have presented in this work a generalization of the scattering-matrix approach to describe the propagation of electromagnetic waves in periodically patterned multilayer structures containing materials with any kind of optical activity and anisotropy. This generalized formalism enables us to tackle important physical problems that have been traditionally out of the scope of this approach. Thus, for instance, the method can be applied to describe all the basic magneto-optical effects in any possible configuration in magnetic structures. Moreover, the method can also be used to study the wave propagation in periodic structures containing an arbitrary number of birefringent/dichroic layers.

We have illustrated the use and capabilities of the method by analyzing a recent experiment in which the transverse magneto-optical Kerr effect (TMOKE) was investigated in an Fe film with a periodic array of subwavelength holes. ${ }^{14}$ We have shown, in excellent agreement with the experiment, that the TMOKE signal can be resonantly enhanced when the samples are illuminated with an appropriate wavelength, and we have attributed this phenomenon to the excitation of surface plasmon polaritons. This resonant enhancement of the magneto-optical signal is closely related to the phenomenon of extraordinary optical transmission (EOT), ${ }^{39,40}$ which indeed takes place in these perforated Fe films. The systematic analysis of the interplay between the EOT phenomenon and the different magneto-optical effects in perforated magnetic films will be the subject of a forthcoming publication.

\section{ACKNOWLEDGMENTS}

We would like to thank G. Armelles and J. F. Torrado for providing us the experimental data on iron films. G. Armelles also provided the experimental values of the dielectric constants, and E. Ferreiro-Vila performed the Polar Kerr experiments for the Fe film. B.C. and A.G.-M. acknowledge funding from the EU (NMP3-SL2008-214107-Nanomagma), the Spanish MICINN ("MAPS" MAT2011-29194-C02-01, "MAGPLAS" MAT2008-06765C02-01/NAN, and "FUNCOAT" CONSOLIDER INGENIO 2010 CSD2008-00023), and the Comunidad de Madrid ("MICROSERES-CM" S2009/TIC-1476). J.C.C. acknowledges financial support from the Spanish MICINN (Contract No. FIS2011-28851-C02-01) and from the German foundations Carl Zeiss Stiftung and Stifterverband für die Deutsche Wissenschaft via the award of an "InnoLecture-Gastdozentur." 


\section{APPENDIX A: FAST FOURIER FACTORIZATION}

The scattering approach, as formulated in Sec. II, is known to have important convergence problems when metals are involved. These problems are specially pronounced when the structures contain noble metals and the infrared range is investigated. These problems are well known in the theory of gratings, ${ }^{41}$ and it has been understood that they originate from the incorrect factorization of the product of two periodic discontinuous functions. Such a product appears, in particular, in the constitutive relation $\mathbf{D}=\bar{\epsilon} \mathbf{E}$, where $\mathbf{D}$ is the displacement vector. When calculating the Fourier components of $\mathbf{D}$ in Sec. II A, we have used the so-called Laurent's rule. This rule states that the Fourier components $h_{n}$ of the product $h(x)$ of two arbitrary functions $f(x)$ and $g(x)$ are given by

$$
h_{n}=\sum_{m=-\infty}^{\infty} f_{n-m} g_{m} .
$$

Although this result is correct, as long as the sum extends to infinity, it is not always correct when one truncates the series, as we do numerically. This was recognized by $\mathrm{Li},{ }^{42}$ who established the following rules for factorization:

(i) Let $h(x)=f(x) g(x)$ and either $f(x)$ or $g(x)$ be continuous at some $x=x_{0}$. The other quantity may be discontinuous there. Then, Laurent's rule applies, i.e.,

$$
[h]=\llbracket f \rrbracket[g] .
$$

Here, $[g]$ denotes a column vector constructed with, let us say, $N_{G}$ Fourier components $g_{n}$, and by $\llbracket f \rrbracket$ we denote the $N_{G} \times N_{G}$ Toeplitz matrix whose $(n, m)$ entry is $f_{n-m}$.

(ii) Let $h(x)=f(x) g(x)$ and both $f(x)$ and $g(x)$ be discontinuous at some $x=x_{0}$, but the product $f(x) g(x)$ be continuous there. Then, the so-called inverse rule holds, which is given by

$$
[h]=\llbracket \frac{1}{f} \rrbracket^{-1}[g] .
$$

(iii) Let $h(x)=f(x) g(x)$ and both $f(x)$ and $g(x)$ be discontinuous at some $x=x_{0}$ and the product $f(x) g(x)$ be discontinuous there as well. Then, the product of the two functions in Fourier space cannot be formed by either the Laurent's rule or the inverse rule.

Obviously, in our analysis of the Maxwell's equations in Sec. II A, see Eqs. (9)-(11), we are violating these factorization rules. We are simply using the Laurent's rule, although in the interface between different materials we may have concurrent discontinuities in both the permittivity tensor and the electric field, and in some cases the product (the displacement vector) is discontinuous as well. Thus, our goal now is to reformulate the Maxwell equations in momentum space in order to respect the factorization rules stated above. For this purpose, we make use of the so-called fast Fourier factorization put forward by Popov and Nevière in Ref. 43.

For the sake of concreteness, let us consider a twodimensional periodic system consisting of an array of circular holes or circular pillars, as in the structure of Sec. III. Now, let us define a vector with the continuous components of the $\mathbf{E}$ and $\mathbf{D}$ fields, i.e., $\mathbf{G}=\left[E_{t}, D_{n}, E_{z}\right]^{T}$. Here, $E_{t}$ is the tangential component of the electric field in the $x y$ plane, $D_{n}$ is the normal component of the displacement vector in the $x y$ plane, and $E_{z}$ is the $z$ component of the electric field. These three components are continuous in the $x y$ plane when we cross the boundary of a hole (or pillar) and the permittivity tensor undergoes a discontinuity. Now, let us establish the relation between these field components and the three Cartesian components of the electric field $\mathbf{G}=\hat{F} \mathbf{E}$, where $\mathbf{E}=\left[E_{x}, E_{y}, E_{z}\right]^{T}$. There are many possible choices for $\hat{F}$. We choose to express its matrix elements in terms of the polar angle $\phi(x, y)$ defined as $r e^{i \phi(x, y)}=x+i y$. It is straightforward to show that

$$
\hat{F}=\left(\begin{array}{ccc}
-s & c & 0 \\
\epsilon_{x x} c+\epsilon_{y x} s & \epsilon_{x y} c+\epsilon_{y y} s & \epsilon_{x z} c+\epsilon_{y z} s \\
0 & 0 & 1
\end{array}\right)
$$

where $c$ and $s$ are abbreviations for $\cos \phi$ and $\sin \phi$, respectively.

We now define the inverse of this matrix $\hat{C}=\hat{F}^{-1}$. Thus, $\mathbf{E}=\hat{C} \mathbf{G}$. Let us recall the constitutive relation $\mathbf{D}=\bar{\epsilon} \mathbf{E}$, where $\bar{\epsilon}$ is given by Eq. (1). This relation can be now written as

$$
\mathbf{D}=\bar{\epsilon} \hat{C} \cdot \mathbf{G}=\bar{\epsilon} \hat{C} \cdot \hat{F} \mathbf{E},
$$

whose elements are now expressed as the product of a discontinuous function and a continuous one. Thus, using Laurent's rule for the first term of the product and the inverse rule for the second one, the Fourier components of the displacement vector can be calculated as

$$
[\mathbf{D}]=\llbracket \bar{\epsilon} \hat{C} \rrbracket \llbracket \hat{C} \rrbracket^{-1}[\mathbf{E}] .
$$

This indicates that the Toeplitz matrix of the index tensor $\hat{\eta}$ in the formalism of Sec. II has to be calculated as follows:

$$
\llbracket \hat{\hat{\eta}} \rrbracket=\llbracket \hat{C} \rrbracket \llbracket \bar{\epsilon} \hat{C} \rrbracket^{-1} .
$$

This is indeed the only change that we need to introduce in the formalism to improve significantly the convergence in the problematic cases. Notice that in practice this requires the calculation of the Toeplitz matrix of several trigonometric functions, which in general has to be done numerically.

For the sake of completeness, we now provide simplified expressions for $\llbracket \hat{\hat{\eta}} \rrbracket$ in some cases of special interest for us. First, in the case of an isotropic material, for which $\bar{\epsilon}=\epsilon \hat{1}$, it is straightforward to show that Eq. (A7) reduces to ${ }^{44,45}$

$$
\llbracket \hat{\hat{\eta}} \rrbracket=\left(\begin{array}{ccc}
\llbracket \epsilon \rrbracket^{-1}+\llbracket X \rrbracket \llbracket c^{2} \rrbracket & \llbracket X \rrbracket \llbracket c s \rrbracket & 0 \\
\llbracket X \rrbracket \llbracket c s \rrbracket & \llbracket 1 / \epsilon \rrbracket-\llbracket X \rrbracket \llbracket c^{2} \rrbracket & 0 \\
0 & 0 & \llbracket \epsilon \rrbracket^{-1}
\end{array}\right),
$$

where $\llbracket X \rrbracket=\llbracket 1 / \epsilon \rrbracket-\llbracket \epsilon \rrbracket^{-1}$. This requires, in particular, the calculation of the Fourier components of the trigonometric functions $\cos ^{2} \phi$ and $\cos \phi \sin \phi$, which can be easily done numerically. Notice that these components are independent of the wavelength of the light and therefore, they can be calculated once and forever for a given structure. On the other hand, for the description of the TMOKE we have to consider a permittivity tensor given by Eq. (37). In this case, the Fourier 
components of the index tensor are given by

$$
\llbracket \hat{\hat{n}} \rrbracket^{-1}=\left(\begin{array}{ccc}
\llbracket \epsilon \rrbracket+\llbracket Y \rrbracket \llbracket c^{2} \rrbracket & \llbracket Y \rrbracket \llbracket c s \rrbracket & \llbracket \epsilon_{x z} \rrbracket+\llbracket Z^{\prime} \rrbracket \llbracket c^{2} \rrbracket \\
\llbracket Y \rrbracket \llbracket c s \rrbracket & \llbracket 1 / \epsilon \rrbracket^{-1}-\llbracket Y \rrbracket \llbracket c^{2} \rrbracket & \llbracket Z^{\prime} \rrbracket \llbracket c s \rrbracket \\
-\llbracket \epsilon_{x z} \rrbracket-\llbracket Z \rrbracket \llbracket c^{2} \rrbracket & -\llbracket Z \rrbracket \llbracket c s \rrbracket & \llbracket \epsilon \rrbracket+\llbracket W \rrbracket \llbracket c^{2} \rrbracket
\end{array}\right),
$$

where

$$
\begin{aligned}
\llbracket Y \rrbracket & =\llbracket 1 / \epsilon \rrbracket^{-1}-\llbracket \epsilon \rrbracket, \\
\llbracket Z \rrbracket & =\llbracket \epsilon_{x z} / \epsilon \rrbracket \llbracket 1 / \epsilon \rrbracket^{-1}-\llbracket \epsilon_{x z} \rrbracket, \\
\llbracket Z^{\prime} \rrbracket & =\llbracket 1 / \epsilon \rrbracket^{-1} \llbracket \epsilon_{x z} / \epsilon \rrbracket-\llbracket \epsilon_{x z} \rrbracket, \\
\llbracket W \rrbracket & =\llbracket \epsilon_{x z}^{2} / \epsilon \rrbracket-\llbracket \epsilon_{x z} / \epsilon \rrbracket \llbracket 1 / \epsilon \rrbracket^{-1} \llbracket \epsilon_{x z} / \epsilon \rrbracket .
\end{aligned}
$$

Again, one just needs the evaluation of the Fourier components of both $\cos ^{2} \phi$ and $\cos \phi \sin \phi$. It is worth stressing that in the choice of the normal vectors entering in the Fourier factorization there is a freedom that one can use to further improve the convergence of the calculations. For a discussion of this issue, see Ref. 45.

To conclude, we now want to illustrate the convergence of the results using the fast Fourier factorization described in this appendix. In Fig. 6 we show an example of the results obtained for the reflectivity and the TMOKE for the structure studied in Sec. III. In this figure, the different curves correspond to different values of $N_{G}$, which is the number of reciprocal lattice vectors taken into account in the calculations upon setting a high-momentum cutoff. As one can see, it is possible to converge the calculations to a high precision in the whole range of wavelengths. Moreover, the convergence is rapid and uniform. It is important to emphasize that in order to get results of similar quality for this example without the use of the fast Fourier factorization, values of $N_{G}$ even larger than 1000 are required (not shown here).

\section{APPENDIX B: SOLVING THE NONLINEAR EIGENVALUE PROBLEM}

We detail here a simple strategy to solve numerically the nonlinear eigenvalue problem of Eq. (23), which we have found to work without any problem in all the cases that we
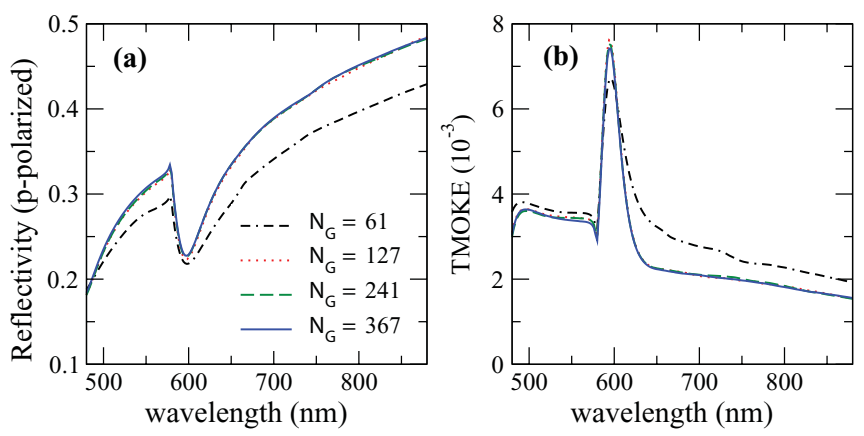

FIG. 6. (Color online) Reflectivity in the absence of magnetic field (a) and TMOKE (b) for the multilayer structure studied in Sec. III for $\theta=25^{\circ}$ and $\varphi=0^{\circ}$ as a function of the wavelength of the incident light. The different curves correspond to results obtained with different numbers of reciprocal lattice vectors $N_{G}$.

have considered. The first step is to multiply both sides of Eq. (23) by $q$ to convert it into the following cubic eigenvalue problem

$$
\left(\mathcal{B}_{3} q^{3}+\mathcal{B}_{2} q^{2}+\mathcal{B}_{1} q+\mathcal{B}_{0}\right) \phi=0,
$$

where we have defined $\mathcal{B}_{n}=\mathcal{A}_{n-1}$. Now, the simplest strategy to solve exactly this cubic problem is to use a standard linearization procedure. ${ }^{46}$ The idea goes as follows: We first define the following vectors

$$
\lambda_{n}=q^{n-1} \phi ; \quad n=1,2,3 .
$$

From this definition, and using Eq. (B1), it is easy to show that the vectors $\lambda_{n}$ satisfy the following equation

$$
\left(\begin{array}{ccc}
0 & 1 & 0 \\
0 & 0 & 1 \\
\mathcal{B}_{0} & \mathcal{B}_{1} & \mathcal{B}_{2}
\end{array}\right)\left(\begin{array}{l}
\lambda_{1} \\
\lambda_{2} \\
\lambda_{3}
\end{array}\right)=q\left(\begin{array}{ccc}
1 & 0 & 0 \\
0 & 1 & 0 \\
0 & 0 & -\mathcal{B}_{3}
\end{array}\right)\left(\begin{array}{l}
\lambda_{1} \\
\lambda_{2} \\
\lambda_{3}
\end{array}\right)
$$

We have thus converted the problem into a generalized linear eigenvalue problem that can be solved with standard linear algebra techniques. The obvious disadvantage of this simple procedure is that one increases the dimension of the problem by a factor of 3 . In this sense, it may be advantageous in some cases to implement other methods like, for instance, the iterative Newton method. In any case, and as illustrated in the previous appendix, we have not found any problem converging the calculations detailed in this work with the linearization procedure, which provides the exact solution of the nonlinear eigenvalue problem.

\section{APPENDIX C: SPATIALLY UNIFORM SLABS}

A multilayer structure may contain some uniform (nonstructured) layers. In particular, this is always the case for the medium of incidence and for the substrate layer. In this sense, it is interesting to discuss how the formalism discussed in Sec. II is simplified in the case of uniform slabs. In this case, the permittivity tensor is diagonal in momentum space: $\left(\hat{\epsilon}_{i j}\right)_{\mathbf{G}, \mathbf{G}^{\prime}}=\tilde{\epsilon}_{i j}(0) \delta_{\mathbf{G}, \mathbf{G}^{\prime}}$, although it can remain fully anisotropic in real space. This implies that all the matrices in momentum representation are also diagonal. The eigenvalue problem of Eq. (23) leads to the following quartic secular equation for $q(\mathbf{G})$ : Focusing on $\mathbf{G}=0$, this equation reads

$$
\sum_{n=0}^{4} D_{n} q^{n}=0
$$

where the coefficients are given by

$$
\begin{aligned}
D_{4}= & \eta_{x x} \eta_{y y}-\eta_{x y} \eta_{y x}, \\
D_{3}= & k_{x}\left[n_{x y} \eta_{y z}+\eta_{y x} \eta_{z y}-n_{y y}\left(\eta_{x z}+\eta_{z x}\right)\right] \\
& +k_{y}\left[n_{y x} \eta_{x z}+\eta_{x y} \eta_{z x}-n_{x x}\left(\eta_{y z}+\eta_{z y}\right)\right],
\end{aligned}
$$




$$
\begin{aligned}
D_{2}= & k_{x}^{2}\left[\eta_{y y}\left(\eta_{x x}+\eta_{z z}\right)-\eta_{x y} \eta_{y x}-\eta_{y z} \eta_{z y}\right] \\
& +k_{y}^{2}\left[\eta_{x x}\left(\eta_{y y}+\eta_{z z}\right)-\eta_{x y} \eta_{y x}-\eta_{x z} \eta_{z x}\right] \\
& +k_{x} k_{y}\left[\eta_{x z}\left(\eta_{y z}+\eta_{z y}\right)+\eta_{y z}\left(\eta_{z x}-\eta_{x z}\right)\right. \\
& \left.-\eta_{z z}\left(\eta_{x y}+\eta_{y x}\right)\right]-\omega^{2}\left(\eta_{x x}+\eta_{y y}\right) \\
D_{1}= & k_{x}^{3}\left[\eta_{x y} \eta_{y z}+\eta_{y x} \eta_{z y}-\eta_{y y}\left(\eta_{x z}+\eta_{z x}\right)\right] \\
& +k_{y}^{3}\left[\eta_{y x} \eta_{x z}+\eta_{x y} \eta_{z x}-\eta_{x x}\left(\eta_{y z}+\eta_{z y}\right)\right] \\
& +k_{x}^{2} k_{y}\left[\eta_{x y} \eta_{z x}+\eta_{x z} \eta_{y x}-\eta_{x x}\left(\eta_{y z}+\eta_{z y}\right)\right] \\
& +k_{y}^{2} k_{x}\left[\eta_{y x} \eta_{z y}+\eta_{y z} \eta_{x y}-\eta_{y y}\left(\eta_{x z}+\eta_{z x}\right)\right] \\
& +\omega^{2}\left[k_{x}^{2}\left(\eta_{x z}+\eta_{z x}\right)+k_{y}^{2}\left(\eta_{y z}+\eta_{z y}\right)\right] \\
D_{0}= & k_{x}^{4}\left(\eta_{y y} \eta_{z z}-\eta_{y z} \eta_{z y}\right)+k_{y}^{4}\left(\eta_{x x} \eta_{z z}-\eta_{x z} \eta_{z x}\right) \\
& +k_{x}^{3} k_{y}\left[\eta_{x z} \eta_{z y}+\eta_{y z} \eta_{z x}-\eta_{z z}\left(\eta_{x y}+\eta_{y x}\right)\right] \\
& +k_{y}^{3} k_{x}\left[\eta_{y z} \eta_{z x}+\eta_{x z} \eta_{z y}-\eta_{z z}\left(\eta_{y x}+\eta_{x y}\right)\right] \\
& +k_{x}^{2} k_{y}^{2}\left[\eta_{z z}\left(\eta_{x x}+\eta_{y y}\right)+\eta_{x y} \eta_{y x}-\eta_{x z} \eta_{z x}-\eta_{y z} \eta_{z y}\right] \\
& +\omega^{2}\left[\omega^{2}-k_{x}^{2}\left(\eta_{y y}+\eta_{z z}\right)-k_{y}^{2}\left(\eta_{x x}+\eta_{z z}\right)\right. \\
& \left.+k_{x} k_{y}\left(\eta_{x y}+\eta_{y x}\right)\right] .
\end{aligned}
$$

For $\mathbf{G} \neq 0$, one just needs to replace $k_{x, y}$ by $k_{x, y}+G_{x, y}$. Equation (C1) has been previously derived (in terms of the components of the permittivity tensor) in the context of the analysis of uniform multilayer structures containing magneto-optical and anisotropic materials. ${ }^{47}$ This equation simplifies in several limiting cases. Thus, for instance, if we consider the typical configuration for measuring the TMOKE, then the permittivity tensor is given by Eq. (37). In this case, $D_{3}=D_{1}=0$ and setting $k_{y}=0$ the solutions of Eq. (C1) are $q_{1}^{2}=\omega^{2} / \eta_{y y}-k_{x}^{2}$ and $q_{2}^{2}=\omega^{2} / \eta_{x x}-k_{x}^{2}$. Moreover, in this case the layer matrix defined in Eq. (29) adopts the following simple form (for $\mathbf{G}=0$ ):

$M=\left(\begin{array}{cccc}\omega^{2} / q_{1} & 0 & -\omega^{2} / q_{1} & 0 \\ 0 & \eta_{x x} q_{2}-\eta_{x z} k_{x} & 0 & -\eta_{x x} q_{2}-\eta_{x z} k_{x} \\ 1 & 0 & 1 & 0 \\ 0 & 1 & 0 & 1\end{array}\right)$.

On the other hand, for an isotropic layer $\bar{\epsilon}=\epsilon \hat{1}$, and in this case $D_{3}=D_{1}=0$ and $q^{2}=\epsilon \omega^{2}-\left(k_{x}^{2}+k_{y}^{2}\right)$. The corresponding layer matrix reads now (for $\mathbf{G}=0$ )

$$
M=\left(\begin{array}{cc}
M_{11} & -M_{11} \\
\hat{1} & \hat{1}
\end{array}\right),
$$

where $\hat{1}$ is the $2 \times 2$ unit matrix and

$$
M_{11}=\frac{1}{q}\left(\begin{array}{cc}
\omega^{2}-k_{y}^{2} \eta & \eta k_{x} k_{y} \\
\eta k_{x} k_{y} & \omega^{2}-k_{x}^{2} \eta
\end{array}\right) .
$$

${ }^{1}$ G. Armelles, A. Cebollada, A. García-Martín, J. M. García-Martín, M. U. González, J. B. González-Díaz, E. Ferreiro-Vila, and J. F. Torrado, J. Opt. A: Pure Appl. Opt. 11, 114023 (2009).

${ }^{2}$ C. Hermann, V. A. Kosobukin, G. Lampel, J. Peretti, V. I. Safarov, and P. Bertrand, Phys. Rev. B 64, 235422 (2001).

${ }^{3}$ J. B. González-Díaz, A. García-Martín, G. Armelles, J. M. GarcíaMartín, C. Clavero, A. Cebollada, R. A. Lukaszew, J. R. Skuza, D. P. Kumah, and R. Clarke, Phys. Rev. B 76, 153402 (2007).

${ }^{4}$ S. Melle, J. L. Menéndez, G. Armelles, D. Navas, M. Vázquez, K. Nielsch, R. B. Wehrspohn, and U. Gösele, Appl. Phys. Lett. 83, 4547 (2003).

${ }^{5}$ J. B. González-Díaz, A. García-Martín, J. M. García-Martín, A. Cebollada, G. Armelles, B. Sepúlveda, Y. Alaverdyan, and M. Käll, Small 4, 202 (2008).

${ }^{6}$ J. B. González-Díaz, B. Sepúlveda, A. García-Martín, and G. Armelles, Appl. Phys. Lett. 97, 043114 (2010).

${ }^{7}$ D. Meneses-Rodríguez, E. Ferreiro-Vila, P. Prieto, J. Anguita, M. U. González, J. M. García-Martín, A. Cebollada, A. GarcíaMartín, and G. Armelles, Small 7, 3317 (2011).

${ }^{8}$ J. Chen, P. Albella, Z. Pirzadeh, P. Alonso-González, F. Huth, S. Bonetti, V. Bonanni, J. Åkerman, J. Nogués, P. Vavassori, A. Dmitriev, J. Aizpurua, and R. Hillenbrand, Small 7, 2341 (2011). ${ }^{9}$ P. K. Jain, Y. Xiao, R. Walsworth, and A. E. Cohen, Nano Lett. 9 , 1644 (2009).

${ }^{10}$ L. Wang, C. Clavero, Z. Huba, K. J. Carroll, E. E. Carpenter,

D. Gu, and R. A. Lukaszew, Nano Lett. 11, 1237 (2011).
${ }^{11}$ M. Diwekar, V. Kamaev, J. Shi, and Z. V. Vardeny, Appl. Phys. Lett. 84, 3112 (2004).

${ }^{12}$ E. T. Papaioannou, V. Kapaklis, P. Patoka, M. Giersig, P. Fumagalli, A. García-Martín, E. Ferreiro-Vila, and G. Ctistis, Phys. Rev. B 81, 054424 (2010).

${ }^{13}$ G. Ctistis, E. T. Papaioannou, P. Patoka, J. Gutek, P. Fumagalli, and M. Giersig, Nano Lett. 9, 1 (2009).

${ }^{14}$ J. F. Torrado, E. Th. Papaioannou, G. Ctistis, P. Patoka, M. Giersig, G. Armelles, and A. García-Martín, Phys. Status Solidi RRL 4, 271 (2010).

${ }^{15}$ A. A. Grunin, A. G. Zhdanov, A. A. Ezhov, E. A. Ganshina, and A. A. Fedyanin, Appl. Phys. Lett. 97, 261908 (2010).

${ }^{16}$ J. F. Torrado, J. B. González-Díaz, G. Armelles, A. García-Martín, A. Altube, M. López-García, J. F. Galisteo-López, A. Blanco, and C. López, Appl. Phys. Lett. 99, 193109 (2011).

${ }^{17}$ E. Th. Papaioannou, V. Kapaklis, E. Melander, B. Hjörvarsson, S. D. Pappas, P. Patoka, M. Giersig, P. Fumagalli, A. García-Martín, and G. Ctistis, Opt. Express 19, 23867 (2011).

${ }^{18}$ A. Battula, S. Chen, Y. Lu, R. J. Knize, and K. Reinhardt, Opt. Lett. 32, 2692 (2007).

${ }^{19}$ Y. M. Strelniker and D. J. Bergman, Phys. Rev. B 77, 205113 (2008).

${ }^{20}$ V. I. Belotelov, L. L. Doskolovich, and A. K. Zvezdin, Phys. Rev. Lett. 98, 077401 (2007).

${ }^{21}$ G. A. Wurtz, W. Hendren, R. Pollard, R. Atkinson, L. Le Guyader, A. Kirilyuk, Th. Rasing, I. I. Smolyaninov, and A. V. Zayats, New J. Phys. 10, 105012 (2008). 
${ }^{22}$ V. I. Belotelov, I. A. Akimov, M. Pohl, V. A. Kotov, S. Kasture, A. S. Vengurlekar, A. V. Gopal, D. R. Yakovlev, A. K. Zvezdin, and M. Bayer, Nat. Nanotechnol. 6, 370 (2011).

${ }^{23}$ D. Martín-Becerra, J. B. González-Díaz, V. V. Temnov, A. Cebollada, G. Armelles, T. Thomay, A. Leitenstorfer, R. Bratschitsch, A. García-Martín, and M. U. González, Appl. Phys. Lett. 97, 183114 (2010).

${ }^{24}$ J. F. Torrado, J. B. González-Díaz, M. U. González, A. GarcíaMartín, and G. Armelles, Opt. Express 18, 15635 (2010).

${ }^{25}$ V. V. Temnov, G. Armelles, U. Woggon, D. Guzatov, A. Cebollada, A. García-Martín, J. M. García-Martín, T. Thomay, A. Leitenstorfer, and R. Bratschitsch, Nat. Photonics 4, 107 (2010).

${ }^{26}$ A. B. Khanikaev, A. A. Fedyanin, A. V. Baryshev, M. Inoue, and A. B. Granovsky, Opt. Express 15, 6612 (2007).

${ }^{27}$ V. I. Belotelov, D. A. Bykov, L. L. Doskolovich, A. N. Kalish, and A. K. Zvezdin, J. Opt. Soc. Am. B 26, 1594 (2009).

${ }^{28}$ A. B. Khanikaev, S. H. Mousavi, G. Shvets, and Y. S. Kivshar, Phys. Rev. Lett. 105, 126804 (2010).

${ }^{29}$ D. M. Whittaker and I. S. Culshaw, Phys. Rev. B 60, 2610 (1999).

${ }^{30}$ S. G. Tikhodeev, A. L. Yablonskii, E. A. Muljarov, N. A. Gippius, and T. Ishihara, Phys. Rev. B 66, 045102 (2002).

${ }^{31}$ N. Anttu and H. Q. Xu, Phys. Rev. B 83, 165431 (2011).

${ }^{32}$ A. García-Martín, G. Armelles, and S. Pereira, Phys. Rev. B 71, 205116 (2005).

${ }^{33}$ M. Liscidini, D. Gerace, L. C. Andreani, and J. E. Sipe, Phys. Rev. B 77, 035324 (2008).
${ }^{34}$ A. K. Zvezdin and V. Kotov, Modern Magnetooptics and Magnetooptical Materials (IOP, Bristol, 1997).

${ }^{35}$ E. Ferreiro-Vila, J. B. González-Diaz, R. Fermento, M. U. González, A. García-Martín, J. M. García-Martín, A. Cebollada, G. Armelles, D. Meneses-Rodriguez, and E. M. Sandoval, Phys. Rev. B 80, 125132 (2009).

${ }^{36}$ M. Plihal and A. A. Maradudin, Phys. Rev. B 44, 8565 (1991).

${ }^{37}$ Let us stress that $\varphi$ is the angle by which the sample is rotated about the $z$-axis, see Fig. 1(a), while the plane of incidence and the magnetic field are kept fixed and perpendicular to each other.

${ }^{38}$ H. Raether, Surface Plasmons on Smooth and Rough Surfaces and on Gratings (Springer-Verlag, Berlin, 1988).

${ }^{39}$ T. W. Ebbesen, H. J. Lezec, H. F. Ghaemi, T. Thio, and P. A. Wolff, Nature (London) 391, 667 (1998).

${ }^{40}$ F. J. García-Vidal, L. Martín-Moreno, T. W. Ebbesen, and L. Kuipers, Rev. Mod. Phys. 82, 729 (2010).

${ }^{41}$ M. Nevière and E. Popov, Light Propagation in Periodic Media: Differential Theory and Design (Marcel Dekker, New York, 2003).

${ }^{42}$ L. Li, J. Opt. Soc. Am. A 13, 1870 (1996).

${ }^{43}$ E. Popov and M. Nevière, J. Opt. Soc. Am. A 18, 2886 (2001).

${ }^{44}$ A. David, H. Benisty, and C. Weisbuch, Phys. Rev. B 73, 075107 (2006).

${ }^{45}$ R. Antos and M. Veis, Opt. Express 18, 27511 (2010).

${ }^{46}$ I. Gohberg, P. Lancaster, and L. Rodman, Matrix Polynomials (Academic Press, New York, 1982).

${ }^{47}$ M. Mansuripur, J. Appl. Phys. 67, 6466 (1990). 